\title{
CortonaOpen3d. Aprender a proyectar in situ en un contexto histórico con la utilización de software opensource
}

Gianluca Emilio Ennio Vita ${ }^{a}$, Irene Ruiz Bazán ${ }^{b}$

aPolitecnico di Milano, gianluca.vita@polimi.it bolitecnico di Torino, irene.ruizbazan@polito.it

\begin{abstract}
In this communication we present the teaching experience carried out during the seven editions of the Workshop COrtonaOPend of the Scuola di Architettura, Urbanistica, Ingegneria delle Costruzioni of the Polytechnic of Milan, the Accademia di Belle Arti di Brera (Milan) and other collaborating entities, that this year reaches its eighth edition in the historic city of Cortona (Arezzo, Italy). In it, two strong teaching ideas are put in the field: the teaching of opensource Blender $3 D$ software as a project design instrument, which is learned at the same time as the cognitive process of developing ideas and the importance of projecting within the same context where the project is carried out, that is, to design "touching" the place. Thus, all the "disadvantages and discomforts" that a priori could consider that involves moving a large group of students to a historic location to learn a computer design program become a key point and a learning strategie.
\end{abstract}

Keywords: Project, blender, cortona, learning, heritage, $3 d$ design

\section{Resumen}

En esta comunicación presentamos la experiencia docente realizada durante las siete ediciones realizadas del Workshop COrtonaOPend de la Scuola di Architettura, Urbanistica, Ingegneria delle Costruzioni del Politécnico de Milán, de la Accademia di Belle Arti di Brera (Milán) y otras entidades colaboradoras, que este año llega a su octava edición en la ciudad histórica de Cortona (Arezzo, Italia). En el mismo se ponen en campo dos fuertes ideas docentes: la enseñanza del software opensource Blender $3 D$ como instrumento de proyecto, que se aprende contemporáneamente al proceso cognitivo de desarrollo de las ideas y la importancia de proyectar dentro del mismo contexto donde se realiza el proyecto, es decir, de proyectar "tocando" el lugar. Así, todas las "desventajas e incomodidades" que a priori podría considerar que conlleva el trasladar a un numeroso grupo de estudiantes a una localidad histórica para aprender un programa de diseño por ordenador se convierten en puntos clave y estrategias de aprendizaje.

Palabras clave: proyecto, blender, cortona, aprendizaje, patrimonio, diseño tridimensional 
CortonaOpen3d. Aprender a proyectar in situ en un contexto histórico con la utilización de software opensource

\section{Introducción}

Desde el año 2012 y con cadencia anual, se organiza en la ciudad histórica de Cortona situada en la provincia de Arezzo, en la Toscana Italiana. el workshop COrtonaOPen3D. En el mismo, un número variable de alumnos, aproximadamente 50 participantes de diferentes procedencias: la Escuela de Arquitectura del Politécnico de Milán, la Facultad de Bellas Artes de la Accademia di Brera (Milán), la Escuela Superior de Diseño de Aragón o la Universidad de Zaragoza, entre otras, se enfrentan durante diez días al desafío de realizar un proyecto a escala territorial, urbana, arquitectónica o de un objeto de diseño utilizando herramientas de diseño de software libre en un contexto histórico respondiendo a las necesidades planteadas por los ciudadanos de Cortona.

\section{Objetivos}

El objetivo principal del workshop es aprender a proyectar y comunicar una idea utilizando el software opensource en modo consciente y creativo. Esta experiencia se encuadra dentro de una corriente de pensamiento que relaciona directamente la herramienta utilizada para proyectar con el resultado obtenido.

El instrumento utilizado es el software de modelado tridimensional Blender 3D. Los software Open Source son ya una realidad consolidada y fiable con las cuales es posible sustituir o implementar los software "tradicionales" consintiendo a quién los utilice obtener elaborados gráficos de nivel y calidad absolutos sin necesidad de inversiones económicas o seguir prácticas ilícitas, extremo que resulta de fundamental importancia en la formación de futuros jóvenes profesionales que pueden "llevar el software" con ellos a cualquier estudio o contexto laboral donde desarrollen su futura práctica profesional.

El open source es además una filosofía de proyecto que innova y pone en crisis los conceptos tradicionales de propiedad intelectual, instaurando un proceso económico innovador con grandes perspectivas. Son programas que se actualizan y desarrollan entre todos sus usuarios. Open source no significa "trabajar gratis" si no "ganar de forma diferente".

Por otro lado, y no menos importante, se trata además de que el alumno entienda la importancia de conocer, de respirar, de tocar, el lugar donde va a desarrollar su proyecto. Por ello, durante todo el workshop, el estudiante se convierte en un habitante más de la ciudad, tanto porque se aloja en la misma porque desde la organización se plantea el uso de diferentes espacios que normalmente no están dedicados a la docencia, para desarrollar los cursos. Esto permite al alumno permite experimentar, de forma temporal, algunos de los "problemas" que plantea la ciudad de Cortona, como su difícil orografía (en lo alto de una montaña, rodeada todavía por las murallas de origen etrusco), la desbordante presión turística, o la falta de conexión con el territorio y las fracciones circunstantes. Cortona es el municipio con la cuarta mayor extensión en la Toscana, el segundo ayuntamiento italiano que sin ser capital de provincia tiene mayor amplitud territorial. 


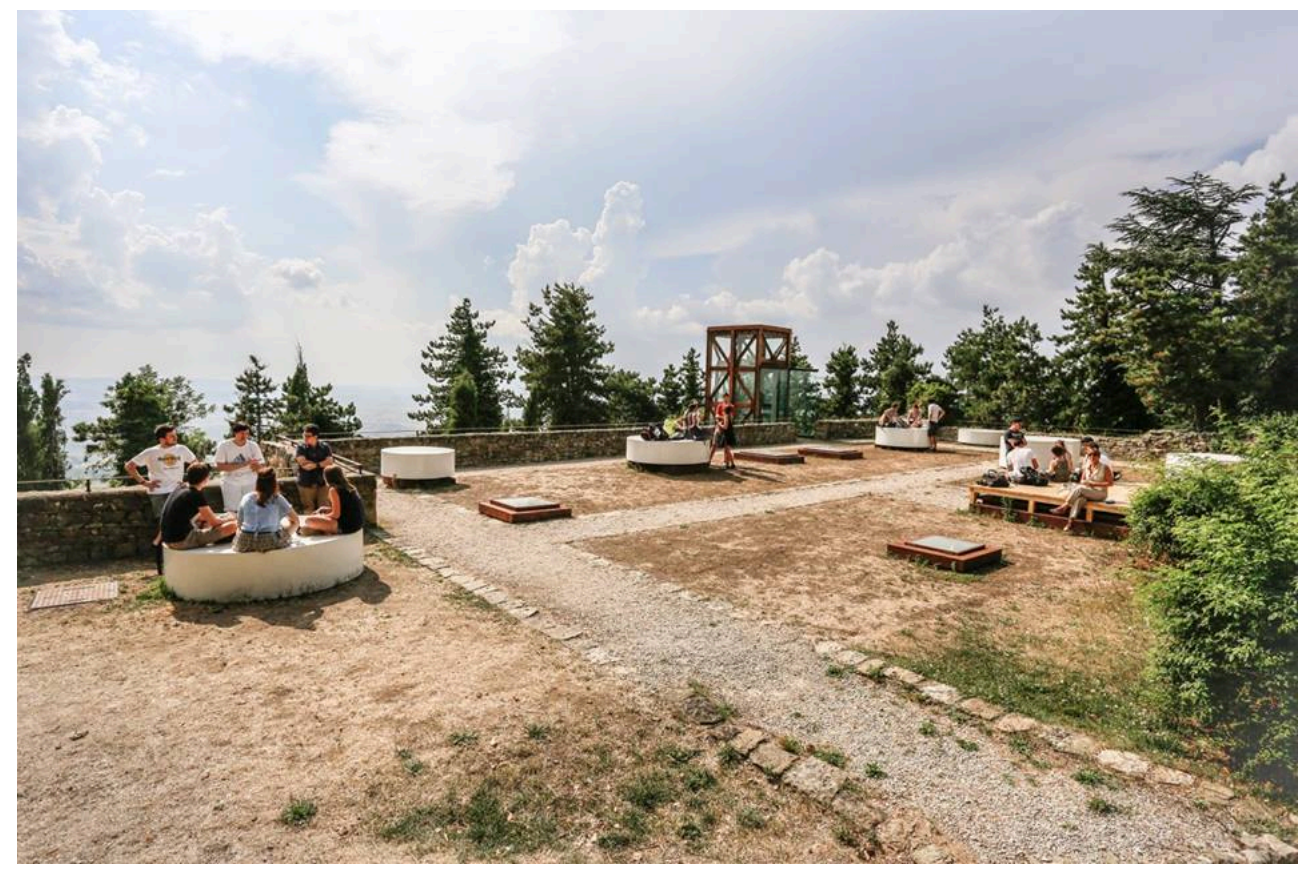

Fig. 1 Estudiantes trabajando en la Fortaleza del Girifalco (Cortona)- Fotografia de los autores

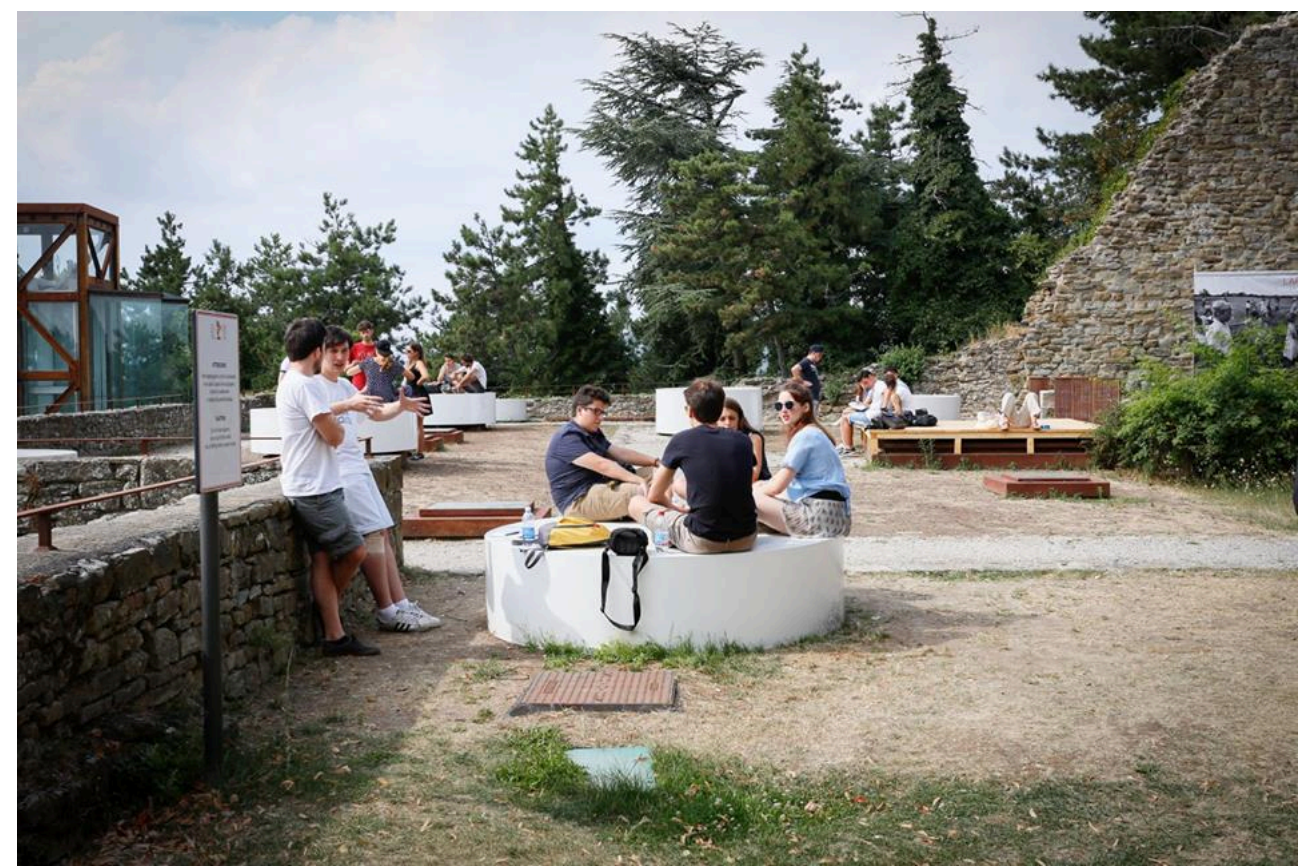

Fig. 2 Estudiantes trabajando en la Fortaleza del Girifalco (Cortona). Fotografia de los autores

El objetivo principal del workshop es por tanto aprender a utilizar el software de diseño tridimensional de una forma diferente y mucho más eficaz a la habitual retahíla de comandos y ejercicios anodinos en la que se convierten la mayor parte de estos cursos que 
CortonaOpen3d. Aprender a proyectar in situ en un contexto histórico con la utilización de software opensource

en muchas ocasiones son vistos casi como accesorios a la formación de los alumnos en las diferentes escuelas de arquitectura, diseño y bellas artes y que por lo tanto, no se vinculan directamente a las principal actividad en la que estos estudiantes se deben ejercitar: el desarrollo proyecto en todas sus diversas declinaciones, con especial atención a su representación.

\section{Desarrollo de la innovación}

Detràas de esta metodología innovadora se encuentra una filosofía de la enseñanza basada constatación de los cambios que la representación del proyecto de arquitectura ha experimentado, y está todavía experimentando, a causa de la revolución informática —con consecuencias tanto positivas como negativas-, y de la voluntad de profundizar en esta realidad.

Los ensayos de Marsall Mc Luhan (Mc Luhan 1967) sobre la relación entre la comunicación y la sociedad moderna, y los de Douglas C. Hofstader (Hofstadter 1979), profesor de ciencias cognitivas, sobre la posibilidad de pensar con una inteligencia artificial, sirven como base para indagar sobre los modos del acto de proyectar contemporáneo.

Siguiendo esta idea, el instrumento, en este caso el software opensource Blender sería parte integrante del proceso de proyecto, y por lo tanto el proyecto nacería de la interacción entre nuestra mente y los instrumentos - herramientas - que ponemos a su disposición. Dichos instrumentos son divididos por Hofstadter en dos categorías: instrumentos físicos actuativos e instrumentos mentales cognitivos. Se produce una ulterior subdivisión entre instrumentos notacionales e instrumentos configurativos, válida y aplicable a sendas categorías citadas. Son instrumentos actuativos el lapicero, el bolígrafo, los software CAD o la máquina fotográfica, ya que nos permiten actuar y "hacer cosas" que sin ellos no podríamos hacer. Pertenecen, sin embargo, a la categoría de los instrumentos cognitivos las figuras geométricas — como triángulos o círculos-, los diagramas de flujo, las listas... 


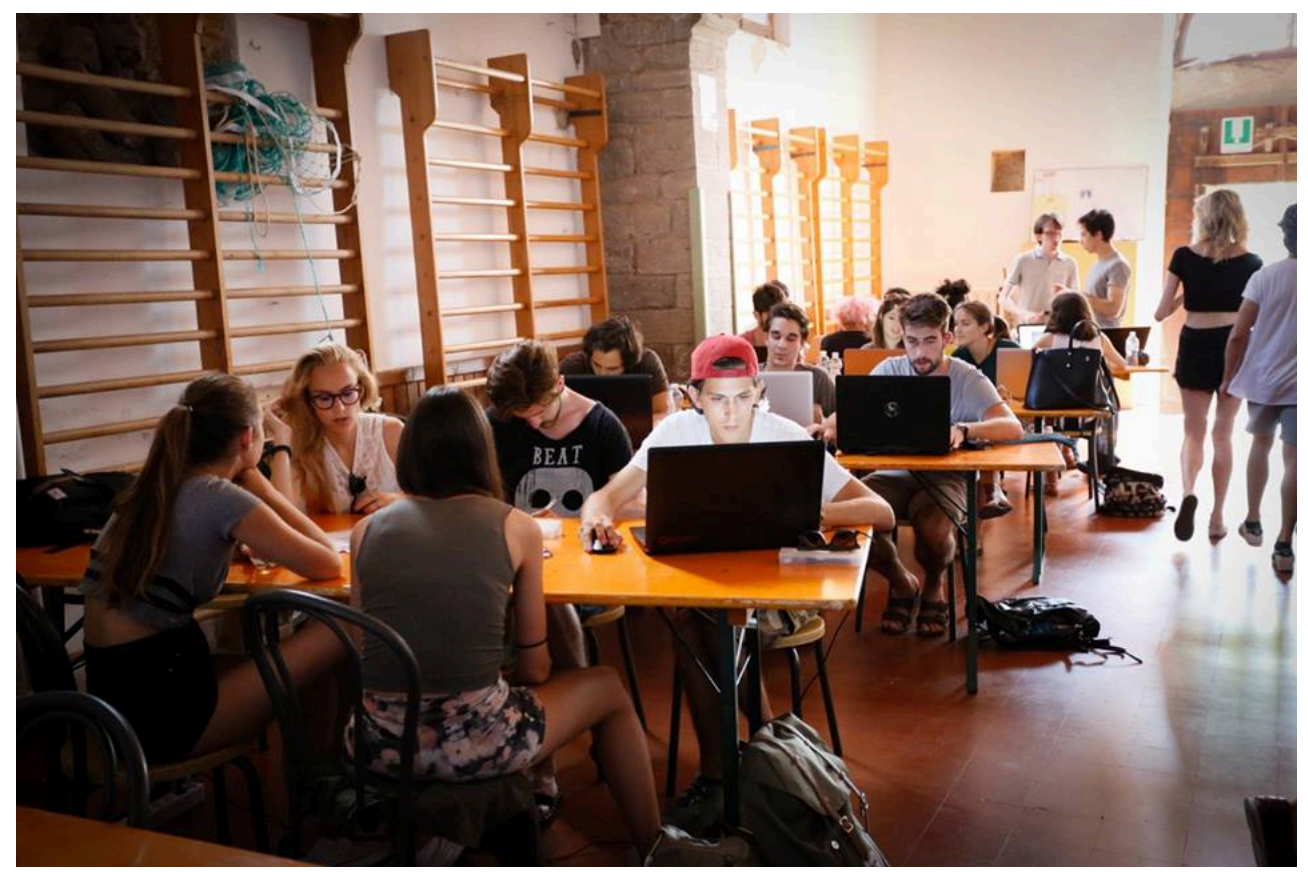

Fig. 3 Estudiantes trabajando en la iglesia de San Sebastiano (Cortona). Fotografia de los autores

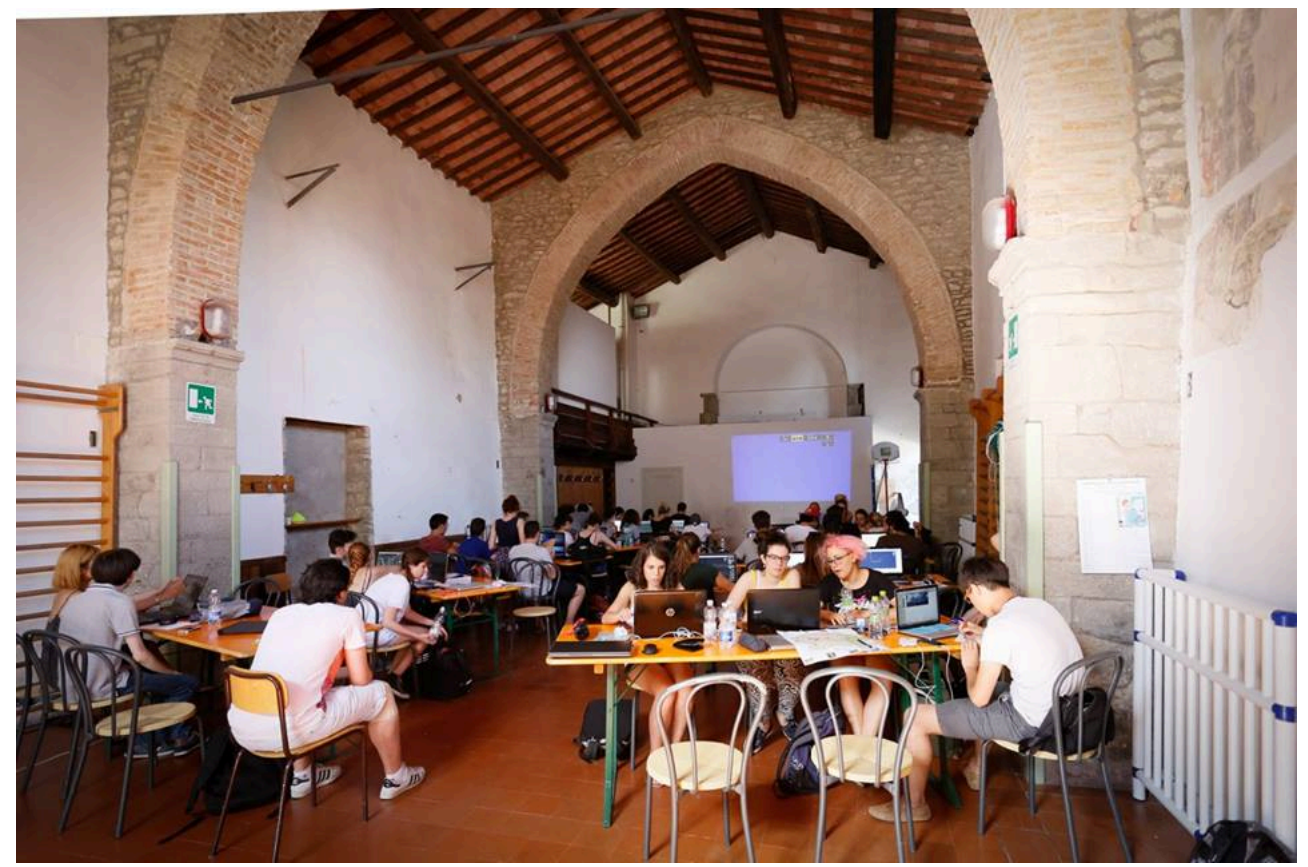

Fig. 4 Estudiantes trabajando en la iglesia de San Sebastiano (Cortona). Fotografia de los autores 
CortonaOpen3d. Aprender a proyectar in situ en un contexto histórico con la utilización de software opensource

Los instrumentos, tanto actuativos como cognitivos, pueden a su vez trabajar en modo notacional o configurativo. Los notacionales trabajan siguiendo esquemas lineales (la lista de la compra, la hoja de cálculo) y permiten dar orden al pensamiento. Los configurativos, por el contrario, permiten a la mente encontrar o producir patrones, estructuras, configuraciones, etc. desde un conjunto no ordenado (la pintura, la adivinación a través de los posos del café o las constelaciones).

Bajo estas dos premisas, se sostiene la hipótesis inicial, sobre la que se basa el desarrollo del programa docente del workshop: el acto de proyectar no es un proceso abstracto: un proyecto no nace sólo en el interior de nuestra mente, sino que se genera por la interacción con las "cosas" que nos circundan.

En el workshop, esta interacción se realiza en dos niveles. De una parte, una vez adquiridas las nociones básicas del software, se comienza a realizar un proyecto, generalmente por grupos de estudiantes de diferente procedencia, y es precisamente durante el desarrollo del proyecto cuando el estudiante aprende y aplica en contemporaneidad las funciones avanzadas del mismo. Este proceso les permite obtener resultados diferentes, por lo general más creativos, que si hubiesen primero "pensado" el proyecto con los instrumentos tradicionales, en dos dimensiones, y después lo hubiesen "extrusionado, elevado..." como se hace convencionalmente, cuando se considera que el software tridimensional sirve solo para desarrollar o representar una idea anterior y no, como intentamos transmitir al alumno, para generarla. A través de la adquisición de un conocimiento y la posibilidad de su aplicación práctica el estudiante adquiere no solo la noción, sino que la utiliza en su proceso cognitivo para proyectar.

Por otra parte, esta interacción "con las cosas" necesaria para el proyecto se produce también por la total inmersión que el estudiante realiza en la ciudad de Cortona.

Durante los primeros días a la introducción al software se acompañan algunas ponencias invitadas de emprendedores locales, representantes políticos y profesores invitados que trasladan a los alumnos las principales inquietudes de Cortona. Por otro lado, los alumnos viven en este contexto histórico donde realizan clases de software, que en los centros docentes tradicionales se suelen realizar en aulas informatizadas, "ocupando" espacios de la ciudad como la biblioteca municipal, antigua iglesia de San Sebastiano (hoy gimnasio de la escuela), el teatro Signorelli o la Fortaleza medicea del Girifalco. La dificultad que esta "ocupación" entraña y los diferentes desplazamientos que los estudiantes deben hacer por la localidad les permiten comprender la dificultad de desarrollar ideas innovadoras en un contexto histórico, en el que para más ende, casi no hay cobertura de datos móviles.

A lo largo de las siete ediciones anteriores se han propuesto diferentes ideas, temas y áreas de proyecto siempre relacionadas con los amplios conceptos de la Smart City, la gestión del patrimonio cultural de la ciudad, la movilidad, etc. referidos a la ciudad de Cortona, para lo que se han implementado clases frontales que introducen estos conceptos. 


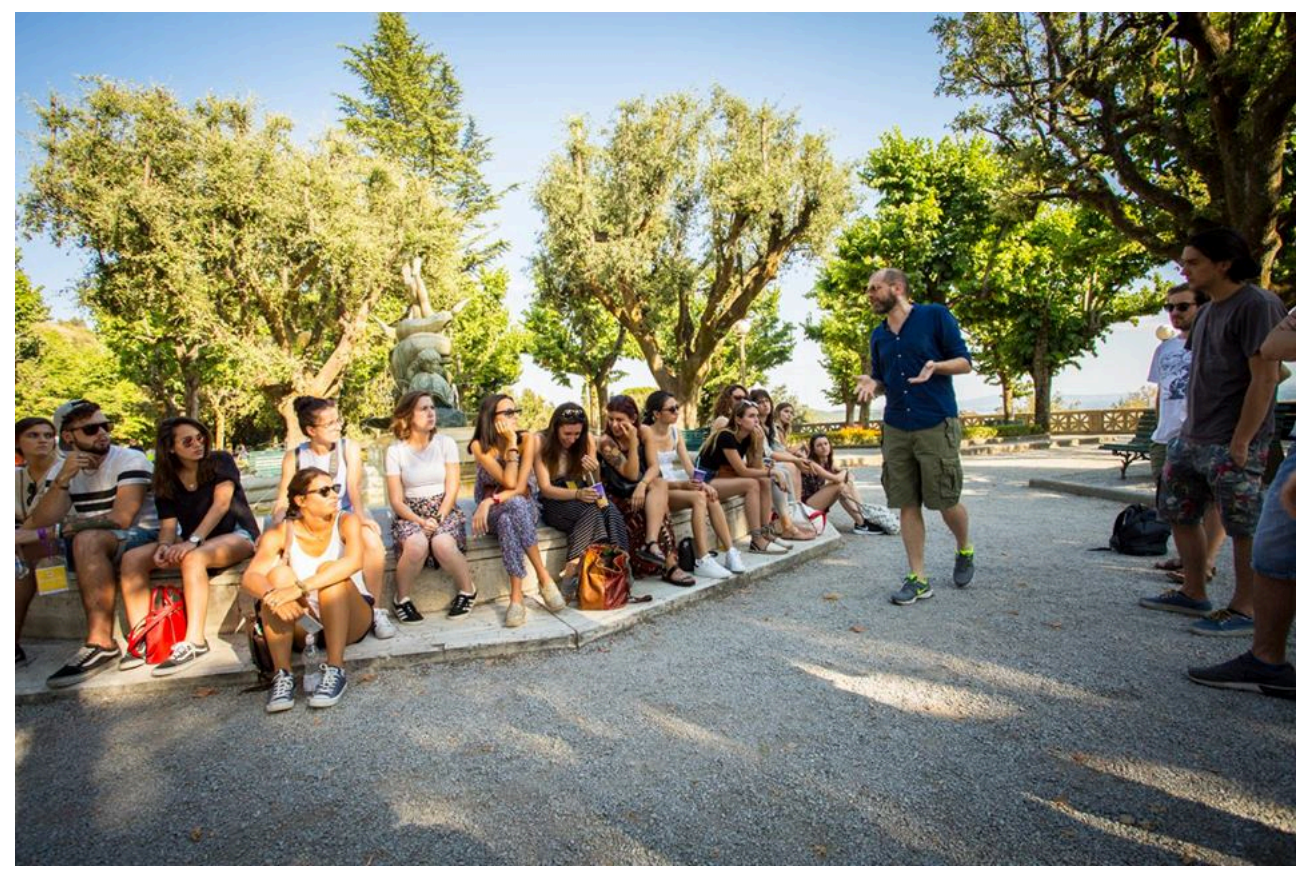

Fig. 5. Lecciones frontales en el Parterre (Cortona). Fotografia de los autores

El programa del curso inicial de software que los alumnos realizan mientras debaten en grupo las ideas del proyecto es el siguiente:

Introducción al software y modelación: Se presenta e instala el software Blender. Se explica la interfaz y los principios de funcionamiento del mismo. Se pasa inmediatamente a la manipulación de objetos. El objetivo es realizar modelos tridimensionales precisos y veloces partiendo de un diseño .dwg o creando objetos directamente dentro de Blender.

Materiales y texturas: A los objetos modelados se les aplica un "shader" (normalmente llamado "material") que imita la reacción a la luz de los objetos... Es importante comprender la naturaleza de los materiales y su funcionamiento para evitar perder tiempo en detalles y complicaciones inútiles y para poder llegar a tener un estilo propio. Se trabaja de modo realístico o gráfico según las preferencias, las situaciones y el resultado que se quiere obtener.

Luz e iluminación: El objeto o proyecto modelado se "pone en escena" con técnicas virtuales similares a las usadas por los fotógrafos en la realidad. Se crea una escena y se posicionan las luces imitando situaciones de interior o exterior, como sucede en el cine o en un estudio fotográfico. Se enseña a los alumnos a elegir entre los diversos tipos de luces existentes y dos configuraciones estándar para render de interior y exterior.

Cámara, fotografía y render: Conseguir un buen render depende en gran parte de la elección de un buen encuadre. Tanto en la realidad como en el espacio virtual depende en gran parte de la elección de un buen encuadre. En ambos casos se trata de "sacar una foto" pero no basta saber cómo hacerlo, hay que desarrollar también cierta sensibilidad por el 
CortonaOpen3d. Aprender a proyectar in situ en un contexto histórico con la utilización de software opensource

"corte" del encuadre. Se explican por tanto algunas reglas fundamentales para realizar un buen encuadre y obtener el mejor render posible.
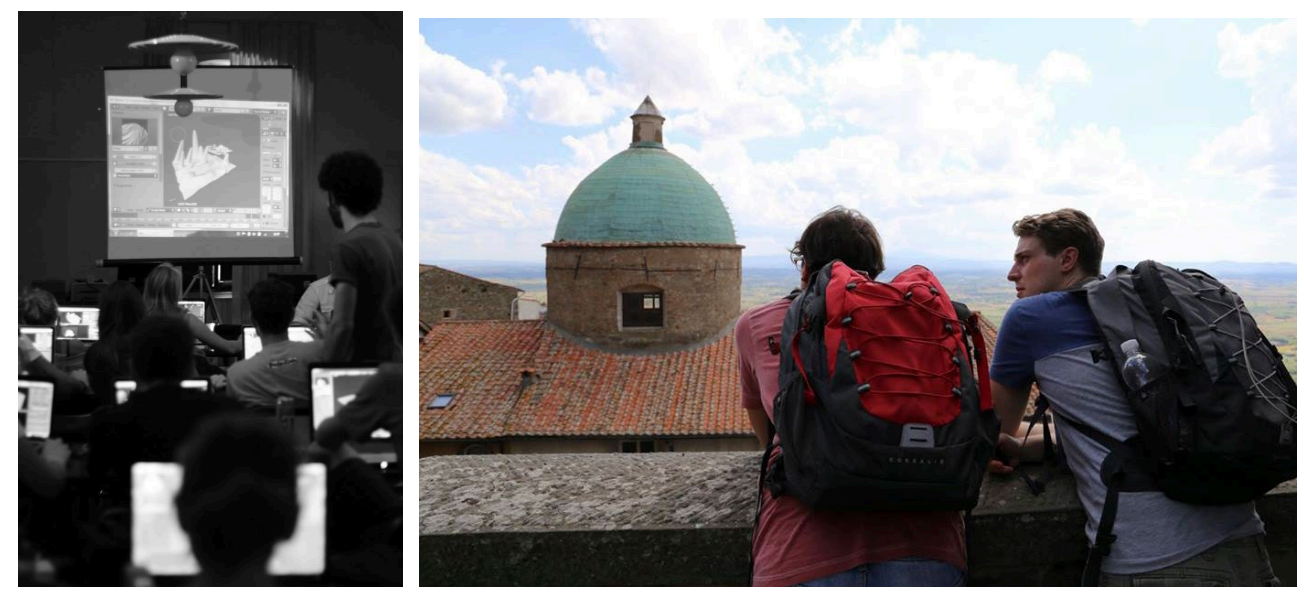

Fig.6 y 7. Lecciones de software y en la propia ciudad de Cortona. Fotografia de los autores

Retoque fotográfico y posproducción: Cuando el render se ha realizado es siempre necesario intervenir para mejorar su aspecto y capacidad comunicativa. Esta operación se puede realizar al interno de Blender o usando un software de retoque digital. Es necesario aprender a mirar de modo objetivo la imagen y comprender qué se puede mejorar y cómo hacerlo. Algunas modificaciones se basan en modificaciones objetivas, otras en la sensibilidad estética de cada uno.

Mientras los alumnos se forman en estos conceptos, se comienza con las tutorías y revisiones de sus ideas del proyecto, de forma que aprendizaje y del software y desarrollo del proyecto van de la mano.

Para motivar a los alumnos a desarrollar sus capacidades, el proyecto se desarrolla en un contexto de "concurso", en el que existen dos premios, el de un jurado compuesto por personalidades locales y universitarias y el de sus propios compañeros, que eligen entre ellos (sin poder votarse a sí mismos) el mejor proyecto.

El ultimo día los estudiantes tienen que presentar su proyecto ante el jurado, sus compañeros y todas las personas de la ciudad de Cortona que quieran participar en un tiempo aproximado de diez a quince minutos, lo que constituye además una excelente oportunidad para ir desarrollando sus capacidades oratorias y de comunicación. 


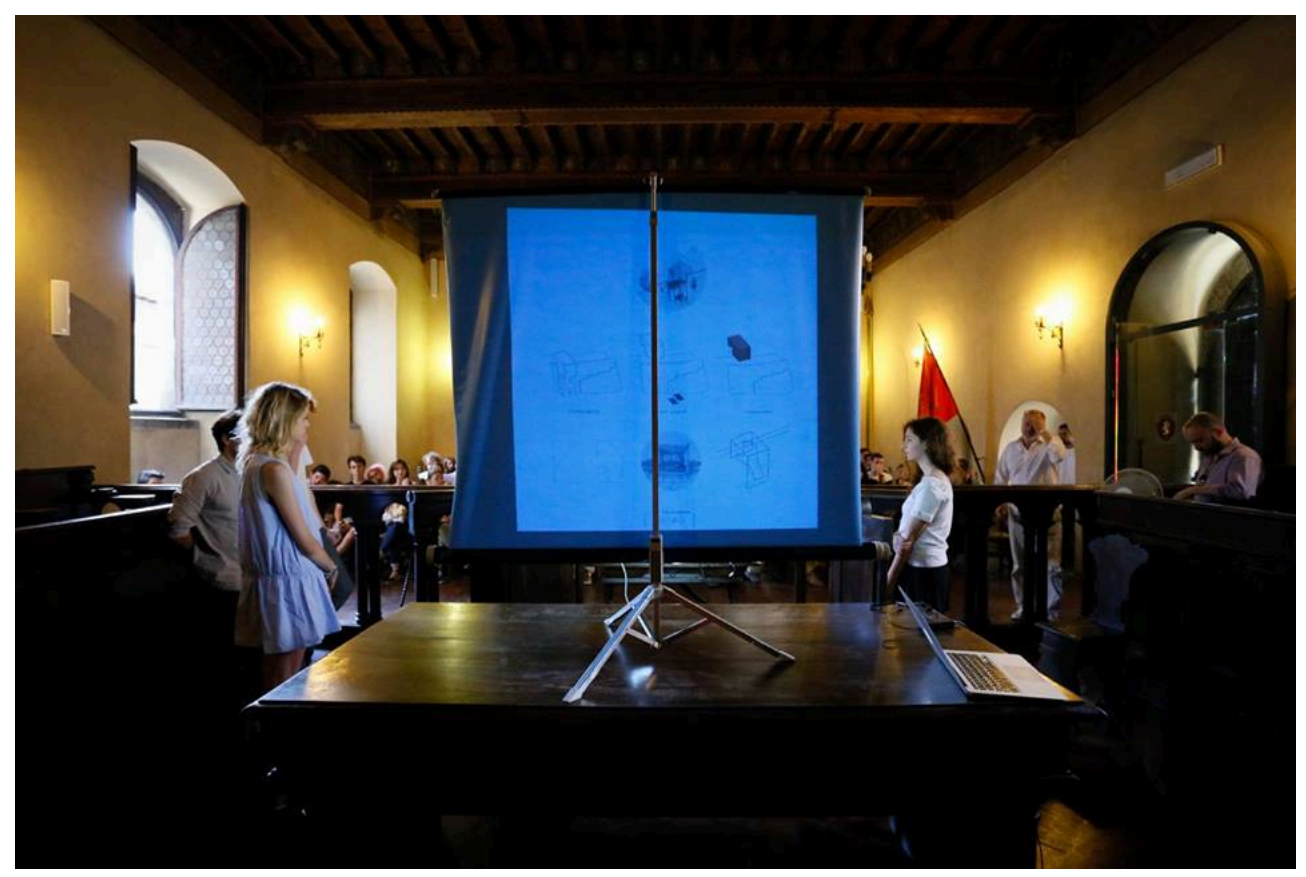

Fig. 8 Presentación final de un grupo. Comune di Cortona (Cortona). Fotografia de los autores

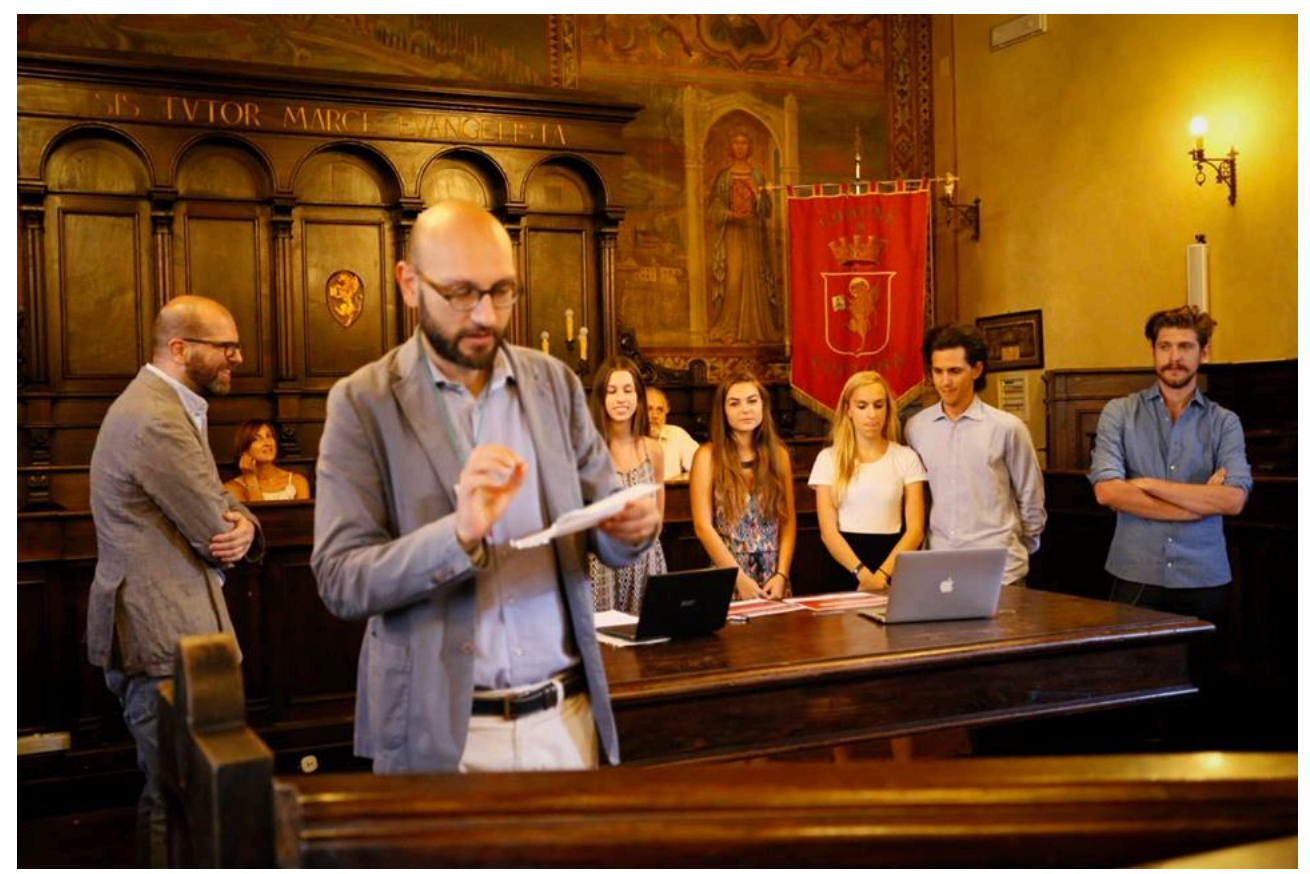

Fig. 9. Premiación final. Comune di Cortona (Cortona). Fotografia de los autores 


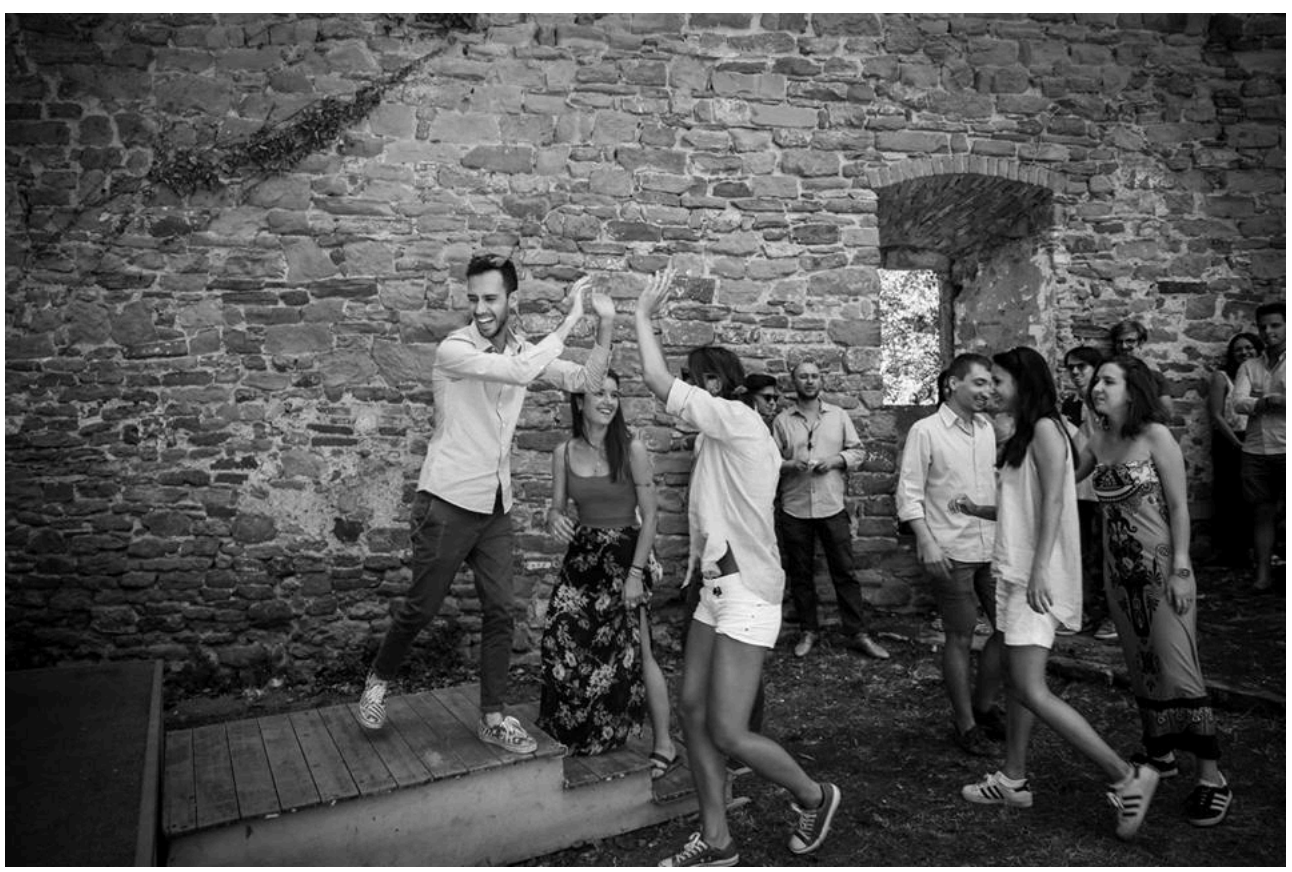

Fig. 10. Premiación final. Fortaleza del Girifalco (Cortona). Fotografía de los autores

\section{Resultados}

La experiencia plurianual del workshop nos ha permitido constatar la efectividad de esta metodología de trabajo. Todos los alumnos que carecían de una base previa en el diseño tridimensional son capaces de realizar un proyecto, a nivel de idea, utilizando Blender y producir al menos, tres render para la presentación final que comuniquen su idea de proyecto.

Por otro lado, se aprecia en todos ellos una mayor sensibilidad en relación al contexto histórico y a sus diferentes problemáticas, desde la conservación a la presión turística, pasando por las oportunidades de comunicación entre visitantes y habitantes o el desarrollo de mejores vías de conexión, física y virtual entre las diferentes fracciones que componen el territorio de la ciudad de Cortona. Se observa además que desarrollan un gran afecto por la localidad que les acoge.

Como tendencias generales, entre los casi cien proyectos realizados en estos años en un contexto como el de Cortona, se observa que los estudiantes tienden a realizar proyectos centrados que más allá de los diferentes temas propuestos cada año, parecen delinear una tendencia transversal común que creemos, están directamente relacionadas con su experiencia de proyectar "fuera del aula":

- Proyectos de desarrollo de los sentidos, especialmente aquellos generalmente menos presentes en los diseños convencionales como el oído, el olfato o tacto.

- Proyectos difusos. Los estudiantes atomizan sus propuestas por toda la ciudad a través de pequeñas intervenciones o de la creación de recorridos. 
- Proyecto de lugares de meditación, observación, relax. El cambio de contexto parece inclinar a los alumnos a realizar proyectos de actuación que promuevan espacios para la reflexión sobre el paisaje, el contexto y el propio ser.

\section{Conclusiones}

A la vista de los proyectos y los resultados obtenidos durante estos años, consideramos el proyecto docente del workshop como un punto clave no sólo en la adquisición de las competencias digitales de diseño del alumno, sino también en su comprensión de las diferentes problemáticas que presenta la realización de un proyecto en un contexto fuertemente connotado como son los centros históricos. Aplicar nuevas formas de concebir el proyecto territorial, urbano, arquitectónico o de diseño a la difícil, y evocadora, realidad de un palimpsesto cultural, social y patrimonial como es Cortona conviviendo entre sus muros con otros estudiantes y con los propios ciudadanos tratando de dar respuesta a las innumerables cuestiones que este lugar plantea sobre sus posibilidades de futuro, se revela una opción innovadora. Aquello que a priori podría resultar incongruente: aprender un potente software de diseño en un lugar que carece de las facilidades y servicios que ofrecen las instalaciones universitarias de las grandes ciudades, ayuda sin embargo a que el esfuerzo y la inmersión realizados marquen de forma significativa en el alumno un antes y un después en su forma de proyectar, en su forma de pensar.

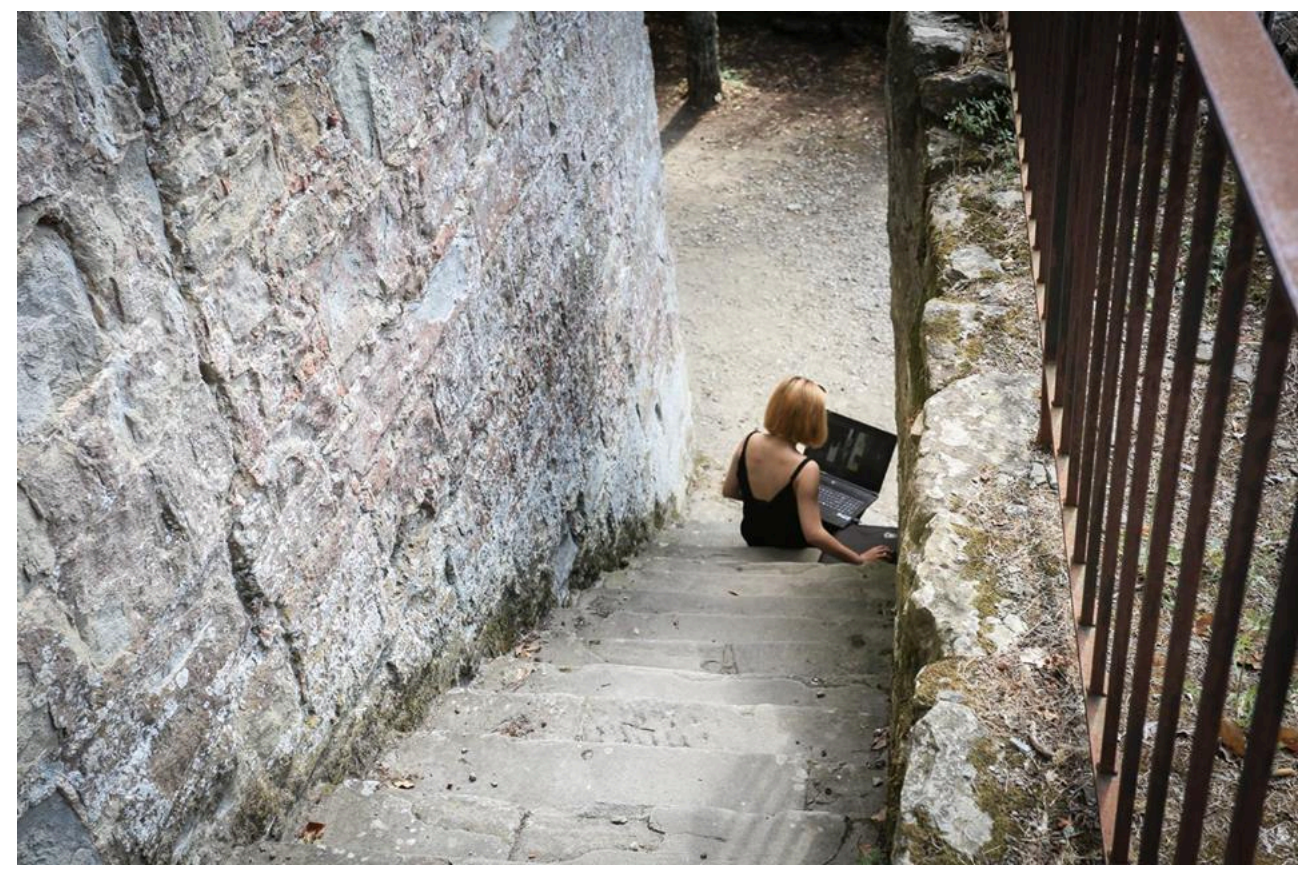

Fig. 11. Desarrollo del workshop. Fortaleza del Girifalco (Cortona). Fotografia de los autores 
CortonaOpen3d. Aprender a proyectar in situ en un contexto histórico con la utilización de software opensource

\section{Referencias}

18ARCHITETTURE. CortonaOpen3d <www.cortonaopen3d.org> [Consulta: 20 marzo 2019]

HOFSTADTER, D.R. (1979). Gödel, Escher, Bach: un'Eterna Ghirlanda Brillante. Milano: Adelphi.

HOFSTADTER, D.R. (1995). Concetti fluidi ed analogie creative. Milano: Adelphi.

MANTOVANI, G. (1990). La qualità dell'interazione uomo compunter. Milano : il Mulino.

McLUHAN, M. (1967). Gli strumenti del comunicare. Milano: Il saggiatore.

ORTEGA, L. (2011). La digitalización toma el mando. Barcelona : Gustavo Gili.

PAPI, D.G., VITA, G.E.E. (2015) "Sistemi SMART per la protezione e la valorizzazione del patirmonio architettonico storico urbano: progetto e rappresentazione". Marmori, A., Puccini, L., Scandellari, V., Van Riel, S., (coord.) En: Architettura e Città. Problemi di conservazione e valorizzazione. Valencia: Altralinea Edizioni. 19-28

ROVETTO, F. (1990). Il piacere di apprendere. Milano: Mondadori

VITA, G.E.E. (2015). "El proyecto Smart City Design como vía de desarrollo de la Ciudad de Cortona" Chaves Martín, M.A.(Ed.) En VII Jornadas Arte y Ciudad. IV Encuentros Internacionales. Ciudad y Comunicación. Madrid: Universidad Complutense de Madrid. 511-518.

VITA, GIANLUCA EMILIO ENNIO "Blender for newcomers, (an architectural desktop?) ". Youtube $<$ https://www.youtube.com/watch? $=$ g_xrV-toaBE $>$ [Consulta: 20 de marzo de 2019] 